\title{
Advantages and pitfalls of elastosonography in the evaluation of cervical lymph nodes
}

\author{
Idil Gunes Tatar, Aydin Kurt, Baki Hekimoglu
}

Department of Radiology, Ankara Diskapi Training and Research Hospital, Turkey

\section{Dear Editor,}

We have read the article recently published in Medical Ultrasonography by Fodor et al with great interest and pleasure [1]. The authors have explicitly presented three cases strikingly demonstrating the utility of elastosonography in clinical practise, correlated with histopathology. The elastosonographic evaluation of a reactive, non-specific lymph node displayed hard areas in less than $50 \%$ of the lymph node. On the other hand elastosonographic evaluation displayed predominantly peripheric stiff areas in more than $50 \%$ of the lymph node in a case of $\mathrm{T}$ cell lymphoma, and hard areas occupying almost entire lymph node was observed in a metastasic lymphadenopathy from a laryngeal carcinoma.

We would like to share a few comments about the advantages and limitations of elastosonography from a radiological point of view. In the last decade many studies have investigated the value of elastosonography for differentiation between benign and malignant cervical lymph nodes [2-4]. Elasticity scores have been widely used based on the percentage of stiff areas within the lymph nodes. For strain ratio measurements, lymph nodes have been evaluated taking the sternocleidomastoid muscle or the connective tissue around the lymph node as the reference.

In one of the pioneer studies on the subject Lyshchik et al published their results in the diagnosis of cervical lymph node metastasis by elastosonography [5]. They

Received 15.09.2013 Accepted 20.09.2013

Med Ultrason

2013, Vol. 15, No 4, 333-334

Corresponding author: Idil Gunes Tatar, M.D.

Department of Radiology,

Ankara Diskapi Training and Research Hospital, 06110, Altındag/ Ankara, Turkey

Phone:+90312 5962616

E-mail: idilttr@yahoo.com measured strain index of the lymph nodes by comparing the absolute strain values of the lymph nodes with the absolute strain values of the nearby muscles. Strain index greater than 1.5 was suggested as the best criterion in discrimination of metastatic lymph nodes with $98 \%$ specificity, $85 \%$ sensitivity, and $92 \%$ accuracy.

Alam et al used a 5-pattern color scoring system based on distribution and percentage of stiff areas in the cervical lymph nodes [6]. The elastosonographic cut-off line for reactive versus metastatic lymph nodes was set between patterns 2 and 3 while patterns 3-5 were considered metastatic. They also evaluated the sum of scores of five gray-scale criteria, ie short-axis diameter, shape, border, echogenicity and presence or absence of hilum. The sonographic cut-off line for reactive versus metastatic was set between scores 6 and 7. Sensitivity, specifity and accuracy of B-mode sonography were $98 \%, 59 \%$ and $84 \%$ respectively; $83 \%, 100 \%$ and $89 \%$ for elastosonography; and $92 \%, 94 \%$ and $93 \%$ for the combined evaluation. Authors proposed that the integrated use of elastosonography and gray-scale sonography has the potential to improve the diagnosis of metastatic enlarged cervical lymph nodes.

In a meta-analysis of the real-time elastosonography for the differentiation of benign and malignant superficial lymph nodes Ying et al. concluded that even though elasticity score measurement had a good diagnostic accuracy it had significant inter-observer variability [7]. They suggested the use of strain ratio measurement was needed for analysis of elasticity of the lymph node. Tan et al. evaluated 128 cervical lymph nodes, 58 of which were malignant, by elastosonography. A strain ratio $>1.5 \mathrm{dem}-$ onstrated $92.5 \%$ sensitivity and $53.4 \%$ specificity [8].

A pitfall of elastosonography is that lymph nodes that are not wholly infiltrated by tumour cause a confusion since elasticity scores or strain ratios evaluate stiffness with reference to the entire nodes. At this point quantitative elastosonography may overcome this disability by 
allowing the evaluation of different parts within lymph nodes. The demonstration of the the areas with increased stiffness may be beneficial to target the sampling by fine needle aspiration to those specific areas inside the lymph node. In this sense Bhatia et al. examined 55 cervical lymph nodes, 31 of which were malignant, by quantitative shear wave elasticity imaging. Authors found out that the median stiffness of malignant nodes, $25.0 \mathrm{kPa}$, was higher than that of benign nodes, $21.4 \mathrm{kPa}$ [9]. Nevertheless the discrimination of shear wave elasticity imaging was low since the optimal cut-off value ( $30.2 \mathrm{kPa})$ had only $41.9 \%$ sensitivity, $100 \%$ specificity and $61.8 \%$ accuracy.

Preliminary evidence suggests that elastosonography may be helpful to discriminate malignant cervical lymph nodes but further research is required to define the elastosonographic criteria which can be used in the diagnostic work-up of cervical lymph nodes.

\section{References}

1. Fodor D, Pascu I, Pop S, Poanta L. The utility of elastography and CEUS for the differentiation between benign and malignant cervical lymphadenopathy. Three cases report. Med Ultrason 2013; 15: 63-66.

2. Kurt A, Gunes Tatar I, Ipek A, Hekimoglu B. B-Mode and Elastosonographic Evaluation to Determine the Reference
Elastosonography Values for Cervical Lymph Nodes. ISRN Radiology Vol. 2013(2013), doi.org/10.5402/2013/895287

3. Teng DK, Wang H, Lin YQ, Sui GQ, Guo F, Sun LN. Value of ultrasound elastography in assessment of enlarged cervical lymphnodes. Asian Pac J Cancer Prev 2012; 13: 20812085.

4. Bhatia KS, Cho CC, Yuen YH, Rasalkar DD, King AD, Ahuja AT. Real-time qualitative ultrasound elastography of cervical lymph nodes in routine clinical practice: interobserver agreement and correlation with malignancy. Ultrasound Med Biol 2010;36:1990-1997.

5. Lyshchik A, Higashi T, Asato R, et al. Cervical lymph node metastases: diagnosis at sonoelastography-initial experience. Radiology 2007; 243: 258-267.

6. Alam F, Naito K, Horiguchi J, Fukuda H, Tachikake T, Ito $\mathrm{K}$. Accuracy of sonographic elastography in the differential diagnosis of enlarged cervical lymph nodes: comparison with conventional B-mode sonography. AJR Am J Roentgenol 2008; 191: 604-610.

7. Ying L, Hou Y, Zheng HM, Lin X, Xie ZL, Hu YP. Real-time elastography for the differentiation of benign and malignant superficial lymph nodes: a meta-analysis. Eur J Radiol 2012; 81: 2576-2584.

8. Tan R, Xiao Y, He Q. Ultrasound elastography: its potential role in assessment of cervical lymphadenopathy. Acad Radiol 2010; 17: 849-855

9. Bhatia KS, Cho CC, Tong CS, Yuen EH, Ahuja AT. Shear Wave elasticity imaging of cervical lymph nodes. Ultrasound Med Biol 2011; 38: 195-201. 